\section{Modelos de assistência obstétrica na Região Sul do Brasil e fatores associados}

\author{
Obstetric care models in the Southern Region of \\ Brazil and associated factors
}

Modelos de asistencia obstétrica en la región sur de Brasil y factores asociados
Manuela Beatriz Velho 1

Odaléa Maria Brüggemann 1

Christine McCourt 2

Silvana Granado Nogueira da Gama 3

Roxana Knobel 1

Annelise de Carvalho Gonçalves 4

Eleonora d'Orsi 1

\section{Resumo}

Os objetivos do estudo foram identificar modelos de assistência obstétrica em gestantes de risco habitual na Região Sul do Brasil, estimar os fatores associados a esses modelos e os desfechos maternos e neonatais. Realizou-se estudo seccional a partir da pesquisa Nascer no Brasil, de base hospitalar, que envolveu puérperas e recém-nascidos. Foram identificadas 2.668 gestantes de risco habitual. Procedeu-se a uma análise exploratória, com a utilização da proporção de práticas por hospital, entre elas o desencadeamento do trabalho de parto, a presença de acompanhante, a cesárea e o contato pele a pele, para a obtenção de modelos de assistência obstétrica denominados Boas Práticas, Intervencionista I e Intervencionista II. Em seguida, realizou-se uma análise inferencial das características associadas. Os resultados mostraram que o acesso ao financiamento público ou privado, os fatores culturais e a atuação dos profissionais de saúde apresentaram associações com os modelos de assistência. A assistência pública apresentou diferentes contextos: um primeiro, alicerçado em politicas públicas e na prática baseada em evidência; um segundo, baseado na intencionalidade pelo parto vaginal, sem considerar os princípios de humanização. Já a assistência privada é padronizada e centrada no profissional médico, com maiores níveis de intervenção. Conclui-se que há predomínio dos modelos de assistência obstétrica intervencionistas na Região Sul do Brasil, uma assistência na contramão das melhores evidências, e que as mulheres assistidas em hospitais públicos possuem mais chance de serem beneficiadas com as boas práticas.

Trabalho de Parto; Cesárea; Assistência Perinatal; Prática Profissional; Prática Clínica Baseada em Evidências

\author{
Correspondência \\ M. B. Velho \\ Rod. Virgílio Várzea 2970, Torre II, apto. 304, Florianópolis, SC \\ 88032-001, Brasil. \\ manuelavelho@hotmail.com \\ 1 Universidade Federal de Santa Catarina, Florianópolis, Brasil. \\ 2 City, University of London, London, U.K. \\ ${ }^{3}$ Escola Nacional de Saúde Pública Sergio Arouca, \\ Fundação Oswaldo Cruz, Rio de Janeiro, Brasil. \\ 4 Universidade Federal do Rio Grande do Sul, Porto Alegre, \\ Brasil.
}




\section{Introdução}

Recomendações para a prática assistencial em saúde baseada nas melhores evidências disponíveis visam a proporcionar uma alta qualidade no atendimento, para o alcance da melhoria da saúde, da segurança, dos custos e dos resultados, incluindo a diminuição da morbidade e da mortalidade dos sujeitos envolvidos 1. São práticas que fundamentam a atuação profissional e incorporam as preferências do cliente à tomada de decisões sobre os seus cuidados e tratamento, em parceria com os profissionais de saúde 2 .

Em obstetrícia, tem-se como marco a recomendação da Organização Mundial da Saúde (OMS) para a atenção ao parto, que deve ser realizado com o mínimo possível de intervenção, resguardando-se a segurança da mãe e do concepto ${ }^{3}$. Essa recomendação foi decorrente da adoção de más práticas obstétricas, difundidas na rotina assistencial, sem o apoio de evidências para o seu uso e que permanecem até os dias atuais. Essas práticas transformaram a assistência obstétrica de um evento com evolução natural e fisiológica em um procedimento marcado pelo excesso de intervenções. Vale destacar que as recomendações clássicas da OMS foram reapresentadas em 2018, com evidências sólidas e rediscussão de condutas, para prover uma assistência de boa qualidade, independente do local e do nível de assistência à saúde 4 .

No Brasil, programas públicos têm seus princípios estabelecidos na humanização da assistência ao nascimento, com o principal objetivo de assegurar um melhor acesso, cobertura e qualidade no acompanhamento do pré-natal, da assistência ao parto e do puerpério às mulheres e aos recémnascidos, na perspectiva dos direitos de cidadania. $\mathrm{O}$ fato é que são encontradas dificuldades para a efetiva implementação dos programas e adequação dos serviços de saúde, que esbarram em questões administrativas, sociais, econômicas e culturais, além de práticas consolidadas, muitas vezes baseadas na conveniência de profissionais e instituições de saúde 5.

Nesse cenário, a organização da assistência obstétrica difere nos diversos contextos assistenciais, com a realização de práticas segundo o modelo de assistência implementado. Para tanto, os objetivos do estudo foram identificar modelos de assistência obstétrica em gestantes de risco habitual na Região Sul do Brasil, estimar os fatores associados a esses modelos e os desfechos maternos e neonatais.

\section{Método}

Os dados analisados foram provenientes da Região Sul, coletados na pesquisa Nascer no Brasil, estudo nacional de base hospitalar que envolveu puérperas e seus recém-nascidos. A determinação do tamanho da amostra no estudo original constituiu-se de três etapas. A primeira etapa considerou elegíveis todos os hospitais com quinhentos ou mais partos/ano em 2007, conforme referência do Sistema de Informações sobre Nascidos Vivos (SINASC). Os hospitais foram estratificados pelas cinco macrorregiões do país, localização (capital ou não capital) e tipo de hospital (privado, público e misto). $\mathrm{Na}$ segunda etapa, foi realizado um método de amostragem inversa, para garantir o número mínimo de sete dias de coleta de dados em cada hospital. A terceira etapa foi composta pelas puérperas hospitalizadas por ocasião do nascimento de seus conceptos vivos, ou mortos com peso ao nascer $\geq 500 \mathrm{~g}$ e/ ou idade gestacional $\geq 22$ semanas.

O cálculo amostral em cada estrato foi determinado a partir da frequência de $46,6 \%$ do desfecho cesárea (dado referente ao ano 2007), com significância de 5\% para detectar diferenças de 14\% entre os tipos de serviço, poder de $95 \%$ e efeito de desenho de 1,3, o que resultou em uma amostra mínima de 450 mulheres por estrato. Participaram do estudo nacional 23.894 mulheres, distribuídas em 266 hospitais, com a realização de 90 entrevistas em cada hospital. O cálculo amostral do estudo nacional permitiu a análise de desfechos com magnitude superior a 3\% em cada região geográfica. Informações adicionais sobre o desenho amostral estão detalhadas em Vasconcellos et al. 6.

A coleta de dados ocorreu por meio de entrevistas face a face com as puérperas, após um período de seis horas do evento do nascimento, durante a internação hospitalar, e também por meio dos prontuários da puérpera e do recém-nascido, bem como por meio de cartões de pré-natal fotografados. Informações sobre a instituição hospitalar foram coletadas em entrevista com o gestor do hospital. 
Informações sobre o pós-parto, por sua vez, foram obtidas por meio de entrevistas telefônicas realizadas com as puérperas, entre o 45o dia e o sexto mês após o nascimento 7 .

Para o presente estudo, utilizou-se a amostra da Região Sul, composta de 4.187 participantes, das quais 2.668 mulheres foram consideradas gestantes de risco habitual, entrevistadas em 46 hospitais entre março e agosto de 2011. Para determinar uma proxy do risco habitual, foram elencados fatores obstétricos e neonatais com o potencial de excluir gestantes com outras patologias prévias e/ou gestacionais não incluídas. A identificação dos critérios de inclusão e exclusão foi baseada nos estudos de Dahlen et al. 8 e de uma análise, já publicada, sobre os dados nacionais da pesquisa Nascer no Brasil 9. Os critérios de inclusão foram: mulheres com idade gestacional entre 37 e 41 semanas, gravidez única, feto em apresentação cefálica, peso ao nascer entre $2.500 \mathrm{~g}$ e $4.499 \mathrm{~g}$. Os critérios de exclusão foram: mulheres com história de diabetes ou hipertensão arterial gestacional ou pré-gestacional e com o resultado positivo para o vírus da imunodeficiência humana. Valores do índice de massa corporal (IMC) não foram considerados na determinação da gestante de risco habitual, por conta do elevado número de perdas sobre as informações biométricas.

Para a identificação dos modelos de assistência obstétrica, realizou-se uma análise exploratória por meio da técnica de agrupamento não hierárquico (cluster), que permite medir a distância das variáveis na busca por uma similaridade entre as observações. Em outras palavras, trata-se de uma análise que busca homogeneidade interna e heterogeneidade externa. As variáveis selecionadas para a elaboração dos agrupamentos foram: trabalho de parto, presença de acompanhante no trabalho de parto e/ ou nascimento, realização de cesárea e contato pele a pele entre mãe e bebê logo após o nascimento. Foram calculadas as proporções dessas variáveis por hospital, para todos os 46 incluídos na amostra. A partir da análise de cluster, foram identificadas três categorias de modelo de assistência obstétrica, denominados modelo com Boas Práticas, Intervencionista I e Intervencionista II, com a apresentação da proporção das práticas em cada modelo e os respectivos intervalos de 95\% de confiança (IC95\%).

Uma análise descritiva foi conduzida para verificar, em cada modelo de assistência obstétrica, a distribuição das variáveis socioeconômicas, demográficas, obstétricas, e também da assistência prénatal, das instituições e da atuação dos profissionais de saúde. Na análise multivariável, foram estimados os fatores associados ao desfecho modelo de assistência obstétrica, por meio da regressão logística multinomial, com utilização da categoria Boas Práticas como referência para verificar associações com as categorias dos modelos de assistência Intervencionista I e II, na investigação de associações com as mesmas variáveis da análise descritiva. A regressão logística multinomial permitiu estimar a odds ratio (OR) bruta, ajustada e seus respectivos IC95\%.

Em seguida, foram apresentadas as frequências dos desfechos maternos e neonatais investigados e as associações com os modelos de assistência obstétrica, agora analisados como variáveis de exposição. A partir da percepção da mulher, os desfechos maternos investigados foram: respeito dos profissionais, privacidade no trabalho de parto e parto, clareza nas explicações, tempo disponível para fazer perguntas, participação nas decisões, violência obstétrica (verbal, psicológica ou física) e satisfação geral com o atendimento. Com exceção do desfecho violência (variável dicotômica), os demais desfechos maternos foram questionados com cinco opções de resposta (péssimo, ruim, regular, bom e excelente) e agrupados em variáveis dicotômicas: não (péssimo, ruim e regular) e sim (bom e excelente). Os desfechos neonatais investigados foram a realização de qualquer manobra de reanimação no recém-nascido (uso de oxigênio inalatório, ventilação com ambu e máscara, entubação orotraqueal, massagem cardíaca ou uso de drogas), internação hospitalar (qualquer forma de internação do recémnascido) e alta em aleitamento materno exclusivo. Uma análise multivariável dos desfechos maternos e neonatais foi realizada por meio da regressão logística, novamente com a verificação da OR bruta, ajustada, bem como seus respectivos IC95\%.

As variáveis construídas a partir do prontuário da mãe, do prontuário do recém-nascido, do cartão de pré-natal e da entrevista com a puérpera na maternidade foram: idade, escolaridade, classe econômica de acordo com a Associação Brasileira de Institutos de Pesquisa de Mercado (ABIPEME), paridade, idade gestacional e fonte de pagamento do nascimento. A partir da entrevista com a puérpera na maternidade, construíram-se as variáveis seguintes: local de realização do pré-natal, preferência inicial pela via de parto, compreensão a partir do pré-natal sobre a segurança do tipo de parto, decisão pela via de parto no final da gestação e atendimento pelo mesmo profissional de saúde no pré-natal e nascimento. A partir da entrevista com o gestor do hospital, construíram-se as seguintes variáveis: 
Hospital Amigo da Criança e Galba de Araújo. As variáveis correspondentes aos desfechos maternos e alta em aleitamento materno exclusivo foram construídas a partir da entrevista com a puérpera por telefone. As demais variáveis de desfecho neonatal, manobras de reanimação no recém-nascido e internação hospitalar foram construídas a partir das informações constantes no prontuário.

Para todas as análises ajustadas, consideraram-se os seguintes como fatores de confusão: idade, escolaridade, classe econômica ABIPEME e paridade. Esses fatores foram determinados a partir da definição conceitual e de análises anteriores dos desfechos estudados 10,11. O software estatístico Stata/SE versão 13 (https://www.sas.com/) foi utilizado para a análise estatística dos dados, tendo em vista a estrutura do plano amostral e os pesos amostrais, elaborados de forma a compensar as perdas de seguimento.

A pesquisa Nascer no Brasil foi norteada pela Resolução no 196/1996, do Conselho Nacional de Saúde, e obteve aprovação do Comitê de Ética em Pesquisa da Escola Nacional de Saúde Pública Sérgio Arouca, Fundação Oswaldo Cruz, sob o parecer no 92/2010. O consentimento de participação dos diretores e das puérperas foi obtido após leitura do Termo de Consentimento Livre e Esclarecido, e a análise de dados respeitou os preceitos éticos da Resolução no 466/2012 do Conselho Nacional de Saúde.

\section{Resultados}

O agrupamento por similaridade da proporção de práticas por hospital, considerando a prevalência na amostra das práticas trabalho de parto (56,6\%), presença do acompanhante no trabalho de parto e/ ou nascimento $(70,2 \%)$, realização de cesárea $(50,5 \%)$ e contato pele a pele $(33 \%)$, permitiu a identificação de três modelos de assistência obstétrica, assim definidos: modelo com Boas Práticas, Intervencionista I e Intervencionista II (Tabela 1 e Figura 1).

O primeiro agrupamento, denominado modelo de assistência obstétrica com Boas Práticas, apresentou uma prevalência de 31,3\% entre as mulheres atendidas. Recebeu essa nomeação por conta de as proporções das práticas identificadas terem sido as mais próximas das recomendadas pelas evidências. O modelo com Boas Práticas caracterizou-se pela maior proporção de trabalho de parto $(69,7 \%)$, presença de acompanhante $(88,1 \%)$ e realização de contato pele a pele $(51,8 \%)$ em comparação com a totalidade da amostra e, ainda, pela menor proporção de cesárea (37,9\%) (Tabela 1 e Figura 1).

O segundo modelo, denominado Intervencionista I, apresentou a maior prevalência entre mulheres atendidas (40\%) e se caracterizou pela proporção de mulheres que entraram em trabalho de parto $(64,4 \%)$ acima da média da amostra, ao passo que a proporção de cesárea (42,7\%) foi abaixo da média. No entanto, práticas consideradas benéficas, como a presença do acompanhante $(35,8 \%)$ e o contato pele a pele $(22,7 \%)$ estiveram abaixo da média da amostra (Tabela 1 e Figura 1).

O terceiro modelo, denominado Intervencionista II (28,6\%), foi o menos prevalente entre as mulheres atendidas e se caracterizou pela baixa proporção de trabalho de parto $(16,1 \%)$ e de contato pele a pele $(24,3 \%)$, com destaque para as expressivas proporções da presença de acompanhante $(95,8 \%)$ e realização de cesárea $(88 \%)$ (Tabela 1 e Figura 1).

$\mathrm{Na}$ análise descritiva, as mulheres de menor escolaridade e de classe econômica mais baixa que realizaram o atendimento público no pré-natal ou nascimento compuseram mais os modelos de assistência com Boas Práticas e Intervencionista I. Por outro lado, as mulheres de maior escolaridade e de mais elevada classe social que realizaram o atendimento no pré-natal ou nascimento com financiamento privado estiveram mais presentes no modelo Intervencionista II (Tabela 2).

$\mathrm{Na}$ assistência pré-natal, apesar da pequena participação do profissional enfermeiro na amostra, sua atuação foi mais frequente no modelo com Boas Práticas, menos no modelo Intervencionista I e, menos ainda, no modelo Intervencionista II. A preferência pelo parto vaginal no início da gestação, a compreensão a partir de informações transmitidas durante o pré-natal de que o parto vaginal é mais seguro e a decisão pelo parto vaginal no final da gestação foram mais presentes nos modelos com Boas Práticas e Intervencionista I. Já no modelo Intervencionista II, foi identificada a igualdade na preferência inicial pelo parto vaginal ou pela cesárea, a compreensão de que a cesárea é mais segura, ou tanto o parto vaginal como a cesárea são seguros para a mãe. Nesse modelo, deu-se a decisão pela cesárea no final da gestação. A maior continuidade no atendimento pelo mesmo profissional de saúde no pré-natal e nascimento foi encontrada no modelo Intervencionista II (Tabela 2). 
Modelos de assistência obstétrica (cluster), segundo proporções de práticas por hospital em gestantes de risco habitual. Região Sul do Brasil, 2011.

\begin{tabular}{|c|c|c|c|c|}
\hline & \multirow{2}{*}{$\begin{array}{l}\text { Prevalência } \\
\text { das práticas na } \\
\text { amostra } \\
(n=2.668)\end{array}$} & \multicolumn{3}{|c|}{ Modelo de assistência obstétrica $(n=46)$} \\
\hline & & $\begin{array}{c}\text { Boas Práticas } \\
(31,3 \%)\end{array}$ & $\begin{array}{c}\text { Intervencionista I } \\
(\mathbf{4 0 , 0 \% )}\end{array}$ & $\begin{array}{c}\text { Intervencionista II } \\
(28,6 \%)\end{array}$ \\
\hline & $\%($ IC95\%) & $\%($ IC95\%) & $\%($ IC95\%) & $\%($ IC95\%) \\
\hline Trabalho de Parto & $56,6(49,8-63,2)$ & $69,7(62,7-76,8)$ & $64,4(57,9-70,9)$ & $16,1(10,3-22,0)$ \\
\hline Acompanhante * & $70,2(60,7-78,3)$ & $88,1(83,3-92,9)$ & $35,8(24,1-47,5)$ & $95,8(92,9-98,7)$ \\
\hline Cesárea & $50,5(44,0-56,9)$ & $37,9(31,5-44,3)$ & $42,7(35,1-50,2)$ & $88,0(82,2-93,8)$ \\
\hline Contato pele a pele & $33,0(26,9-39,8)$ & $51,8(40,1-63,4)$ & $22,7(16,0-29,4)$ & $24,3(10,1-38,4)$ \\
\hline
\end{tabular}

IC95\%: intervalo de 95\% de confiança.

* No trabalho de parto e/ou nascimento.

\section{Figura 1}

Boxplot do modelo de assistência obstétrica (cluster), segundo proporção de práticas por hospital em gestantes de risco habitual. Região Sul do Brasil, $2011(n=46)$.

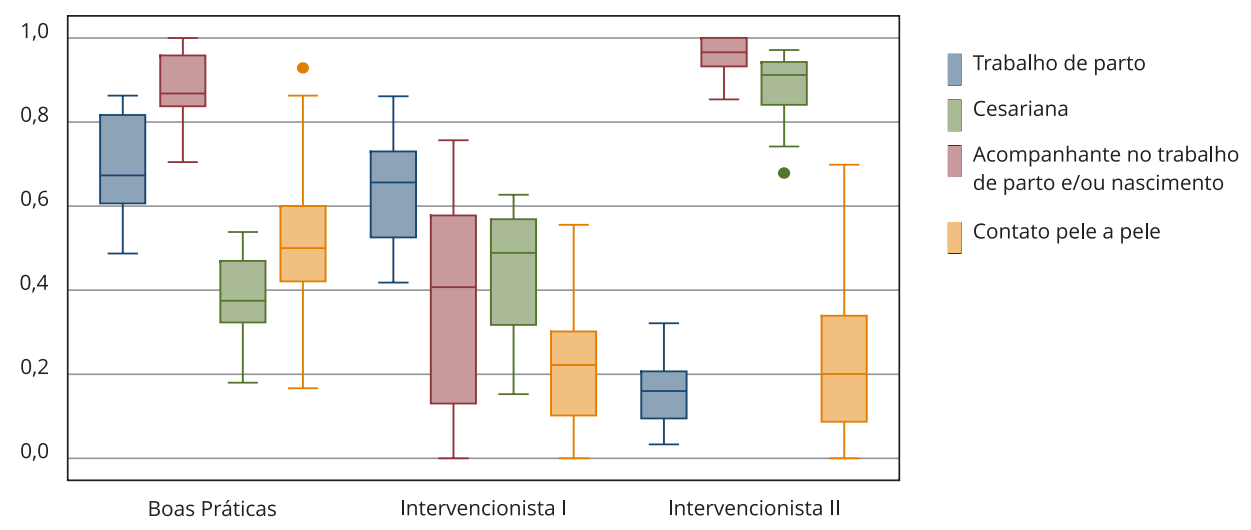

Quanto às características obstétricas, gestações entre 39 e 40 semanas tiveram maior prevalência nos modelos com Boas Práticas e Intervencionista I, ao passo que no modelo Intervencionista II, foi encontrada maior prevalência de gestações entre 37 e 38 semanas. Características institucionais como título de Hospital Amigo da Criança ou o reconhecimento das instituições com o prêmio Galba de Araújo foram mais frequentes no modelo com Boas Práticas, menos no modelo Intervencionista I e inexistentes no modelo Intervencionista II (Tabela 2).

A análise multivariável validou diferenças nos modelos de assistência obstétrica, na identificação dos fatores preditores nos modelos Intervencionistas I e II, comparados com o modelo com Boas Práticas. No modelo Intervencionista I, foram fatores preditores o atendimento na maior parte das consultas de pré-natal por profissional médico e a decisão pela cesárea no final da gestação. No modelo Intervencionista II, foi encontrado um efeito com gradiente positivo no aumento da escolaridade materna, além dos demais fatores preditores identificados na análise ajustada: ter realizado o 
Tabela 2

Distribuição das características na amostra e proporção de mulheres em cada modelo de assistência obstétrica, segundo as variáveis socioeconômicas, demográficas, obstétricas, da assistência pré-natal, das instituições e da atuação dos profissionais de saúde em gestantes de risco habitual. Região Sul do Brasil, $2011(n=2.668)$.

\begin{tabular}{|c|c|c|c|c|}
\hline & \multirow[b]{2}{*}{ Total (\%) } & \multicolumn{3}{|c|}{ Modelo de assistência obstétrica } \\
\hline & & $\begin{array}{l}\text { Boas Práticas } \\
\text { \% (IC95\%) }\end{array}$ & $\begin{array}{l}\text { Intervencionista I } \\
\%(\mathrm{IC} 95 \%)\end{array}$ & $\begin{array}{c}\text { Intervencionista II } \\
\%(\text { IC95\%) }\end{array}$ \\
\hline \multicolumn{5}{|l|}{ Idade (anos) } \\
\hline $12-19$ & $17,8(15,4-20,6)$ & $21,4(18,0-25,1)$ & $19,7(15,7-24,3)$ & $7,0(4,4-11,1)$ \\
\hline $20-34$ & $71,6(69,2-74,0)$ & $69,7(65,3-73,7)$ & $70,4(66,7-73,8)$ & $78,2(73,3-82,4)$ \\
\hline 35 ou mais & $10,6(9,0-12,3)$ & $9,0(7,3-11,1)$ & $10,0(7,9-12,5)$ & $14,8(9,5-22,3)$ \\
\hline \multicolumn{5}{|l|}{ Escolaridade } \\
\hline Ensino Fundamental incompleto & $23,3(19,8-27,2)$ & $28,0(22,0-34,9)$ & $28,2(25,1-31,6)$ & $3,6(1,6-7,9)$ \\
\hline Ensino Fundamental completo & $27,5(24,8-30,4)$ & $31,8(28,0-35,8)$ & $31,2(28,7-33,8)$ & $11,5(8,3-15,6)$ \\
\hline Ensino Médio completo & $37,7(34,4-41,0)$ & $32,8(27,8-38,3)$ & $35,2(32,0-38,5)$ & $52,2(46,1-58,3)$ \\
\hline Ensino Superior completo & $11,5(8,4-15,5)$ & $7,4(5,1-10,7)$ & $5,4(3,5-8,4)$ & $32,7(23,5-43,5)$ \\
\hline \multicolumn{5}{|l|}{ Classe ABIPEME } \\
\hline $\mathrm{D} / \mathrm{E}$ & $9,0(7,2-11,2)$ & $11,9(8,5-16,3)$ & $9,6(7,3-12,7)$ & $2,0(0,4-8,8)$ \\
\hline C & $52,7(48,7-56,7)$ & $57,1(53,0-61,0)$ & $58,9(54,6-63,0)$ & $30,9(19,9-44,6)$ \\
\hline $\mathrm{A} / \mathrm{B}$ & $38,3(33,4-43,5)$ & $31,1(25,2-37,6)$ & $31,5(27,4-35,9)$ & $67,1(50,7-80,2)$ \\
\hline \multicolumn{5}{|l|}{ Paridade } \\
\hline$\geq 1$ & $53,7(50,8-56,7)$ & $54,8(49,6-59,9)$ & $56,3(51,4-61,0)$ & $46,3(42,4-50,2)$ \\
\hline 0 & $46,3(43,3-49,2)$ & $45,2(40,1-50,5)$ & $43,7(39,0-48,6)$ & $53,7(49,8-57,6)$ \\
\hline \multicolumn{5}{|l|}{ Idade gestacional (semanas) } \\
\hline $37-38$ & $37,7(34,2-41,4)$ & $32,9(27,8-38,4)$ & $35,8(31,0-40,9)$ & $51,2(44,2-58,1)$ \\
\hline $39-40$ & $52,7(50,0-55,3)$ & $54,5(50,9-58,0)$ & $53,9(49,5-58,2)$ & $46,6(39,8-53,5)$ \\
\hline 41 & $9,6(9,6-11,6)$ & $12,6(9,5-16,6)$ & $10,3(8,4-12,5)$ & $2,2(1,6-3,1)$ \\
\hline \multicolumn{5}{|l|}{ Local de realização do pré-natal } \\
\hline Serviço público & $62,2(54,1-69,8)$ & $72,6(66,3-78,2)$ & $76,2(68,3-82,7)$ & $12,2(2,2-46,4)$ \\
\hline $\begin{array}{l}\text { Serviço particular ou plano de } \\
\text { saúde }\end{array}$ & $33,6(26,2-41,9)$ & $22,1(17,3-27,7)$ & $20,3(13,7-29,0)$ & $84,5(55,4-96,0)$ \\
\hline Serviço público e particular & $4,2(3,2-5,4)$ & $5,3(3,5-8,1)$ & $3,5(2,1-5,8)$ & $3,4(2,2-5,1)$ \\
\hline \multicolumn{5}{|l|}{$\begin{array}{l}\text { Profissional que atendeu a maior } \\
\text { parte das consultas de pré-natal }\end{array}$} \\
\hline Enfermeiro & $5,0(3,3-7,4)$ & $8,6(5,3-13,7)$ & $4,0(2,1-7,3)$ & $0,4(0,1-1,3)$ \\
\hline Médico & $95,1(92,6-96,7)$ & $91,4(86,3-94,7)$ & $96,0(92,7-97,9)$ & $99,6(98,8-99,9)$ \\
\hline \multicolumn{5}{|l|}{ Preferência inicial pela via de parto } \\
\hline Parto vaginal & $64,2(60,5-67,7)$ & $69,8(62,6-76,1)$ & $66,9(63,4-70,2)$ & $47,7(41,5-54,0)$ \\
\hline Cesárea & $29,0(25,7-32,5)$ & $22,9(18,7-27,7)$ & $26,3(23,0-29,9)$ & $46,3(39,7-53,2)$ \\
\hline Não tinha preferência & $6,8(5,1-9,0)$ & $7,3(3,9-13,5)$ & $6,8(5,0-9,3)$ & $5,9(3,3-10,5)$ \\
\hline \multicolumn{5}{|l|}{$\begin{array}{l}\text { Aconselhamento sobre o tipo de } \\
\text { parto no pré-natal }\end{array}$} \\
\hline Parto vaginal é mais seguro & $56,2(52,3-60,2)$ & $60,0(54,3-65,5)$ & $61,6(56,7-66,3)$ & $37,4(28,5-47,3)$ \\
\hline Cesárea é mais segura & $8,6(7,1-10,3)$ & $6,4(4,5-9,0)$ & $7,6(5,8-9,9)$ & $14,9(10,5-20,9)$ \\
\hline Ambos são seguros & $27,2(23,9-30,8)$ & $25,7(22,1-29,8)$ & $22,3(18,3-26,9)$ & $40,8(30,1-52,5)$ \\
\hline Não ficou esclarecida & $8,0(6,1-10,3)$ & $7,9(5,5-11,3)$ & $8,5(5,1-13,8)$ & $6,8(3,7-12,2)$ \\
\hline \multicolumn{5}{|l|}{$\begin{array}{l}\text { Decisão pela via de parto no final da } \\
\text { gestação }\end{array}$} \\
\hline Parto vaginal & $48,0(42,6-53,5)$ & $59,2(51,2-66,8)$ & $50,2(44,1-56,2)$ & $22,3(15,0-31,9)$ \\
\hline Cesárea & $32,7(26,7-39,2)$ & $18,7(13,3-25,8)$ & $27,5(22,3-33,4)$ & $70,1(57,5-80,2)$ \\
\hline Não tinha preferência & $19,3(15,8-23,4)$ & $22,0(15,2-30,7)$ & $22,3(17,1-28,6)$ & $7,6(4,5-12,6)$ \\
\hline
\end{tabular}

(continua) 


\begin{tabular}{|c|c|c|c|c|}
\hline & \multirow[b]{2}{*}{ Total (\%) } & \multicolumn{3}{|c|}{ Modelo de assistência obstétrica } \\
\hline & & $\begin{array}{c}\text { Boas Práticas } \\
\% \text { (IC95\%) }\end{array}$ & $\begin{array}{c}\text { Intervencionista I } \\
\%(\mathrm{IC} 95 \%)\end{array}$ & $\begin{array}{c}\text { Intervencionista II } \\
\%(\text { IC95\%) }\end{array}$ \\
\hline \multicolumn{5}{|c|}{ Fonte de pagamento do nascimento } \\
\hline Pública & $74,4(64,0-82,6)$ & $90,3(81,5-95,2)$ & $89,6(82,3-94,2)$ & $10,7(1,3-52,3)$ \\
\hline Privada & $25,6(17,4-36,0)$ & $9,7(4,8-18,5)$ & $10,4(5,8-17,7)$ & $89,3(47,7-98,7)$ \\
\hline \multicolumn{5}{|c|}{ Hospital Amigo da Criança } \\
\hline Não & $53,2(37,4-68,3)$ & $27,0(8,0-61,0)$ & $54,4(29,6-77,2)$ & 100,0 \\
\hline Sim & $46,8(31,7-62,6)$ & $73,0(39,0-92,0)$ & $45,6(22,8-70,4)$ & - \\
\hline \multicolumn{5}{|c|}{ Hospital Galba de Araújo } \\
\hline Não & $95,6(82,7-99,0)$ & $92,7(55,8-99,2)$ & $96,1(72,7-99,6)$ & 100,0 \\
\hline $\operatorname{Sim}$ & $4,4(1,0-17,4)$ & $7,3(0,8-44,2)$ & $3,9(0,4-27,4)$ & - \\
\hline \multicolumn{5}{|c|}{$\begin{array}{l}\text { Mesmo profissional no pré-natal e } \\
\text { nascimento }\end{array}$} \\
\hline Não & $66,7(58,3-74,2)$ & $79,3(67,1-87,7)$ & $77,6(69,1-84,4)$ & $18,9(7,7-39,4)$ \\
\hline Sim & $33,3(25,8-41,7)$ & $20,8(12,3-32,9)$ & $22,4(15,6-31,0)$ & $81,1(60,6-92,3)$ \\
\hline
\end{tabular}

ABIPEME: Associação Brasileira de Institutos de Pesquisa de Mercado; IC95\%: intervalo de 95\% de confiança.

pré-natal em serviço particular ou plano de saúde; ser acompanhada na maior parte das consultas por profissional médico; ter a preferência inicial das mulheres pela cesárea; ter a compreensão, a partir do aconselhamento durante o pré-natal, de que tanto a cesárea como o parto vaginal são seguros para a mãe; ter a decisão pela cesárea no final da gestação; ter a continuidade na assistência, pelo mesmo profissional de saúde, no pré-natal e nascimento; realizar a interrupção da gestação entre 37 e 38 semanas de idade gestacional; utilizar fonte de pagamento privada para a assistência ao nascimento (Tabela 3).

A percepção das mulheres sobre o atendimento foi elevada no quesito satisfação e aspectos relacionados: acima de $85,8 \%$. Mulheres atendidas no modelo Intervencionista II, quando comparadas ao modelo com Boas Práticas, tiveram maior chance de se sentirem respeitadas pelos profissionais e de terem privacidade no trabalho de parto e parto. Além disso, essas mulheres tiveram clareza nas informações e tempo disponível para fazer perguntas, bem como participaram das decisões e, por conta disso, houve satisfação geral com o atendimento. No modelo Intervencionista I, as mulheres tiveram menos chance de se sentirem respeitadas pelos profissionais, na comparação com o modelo de assistência obstétrica com Boas Práticas (Tabela 4). O desfecho violência verbal, psicológica ou física obteve uma prevalência de 4,3\% e não apresentou associação com os modelos de assistência (dados não apresentados nas tabelas).

Entre os desfechos neonatais estudados, um em cada dez recém-nascidos foi reanimado em sala de parto, com associações que mostraram o dobro de chance ou mais de ocorrência nos modelos de assistência Intervencionista I e II. A internação hospitalar do recém-nascido teve uma prevalência de $4,7 \%$ e não esteve associada aos modelos de assistência. Alta em aleitamento materno exclusivo obteve uma prevalência elevada (94\%) e uma associação com menor chance de ocorrência no modelo de assistência Intervencionista II, na comparação com o modelo com Boas Práticas (Tabela 4).

\section{Discussão}

A identificação dos modelos de assistência obstétrica permitiu o reconhecimento de distintas formas de organização e condução da assistência, assim como uma aproximação com o perfil das mulheres atendidas em cada modelo. A escolha das práticas para a elaboração dos modelos, entre elas, trabalho de parto, presença de acompanhante no trabalho de parto e/ou nascimento, cesárea e contato pele a pele, encontra-se fundamentada na prática baseada em evidência ou nos princípios de humanização da assistência. Essas escolhas se devem pela elevada prevalência na realização de cesárea sem trabalho de parto em determinadas instituições, fato que impossibilitou o uso de práticas realizadas durante o 
Tabela 3

Odds ratio (OR) ajustada para o desfecho modelo de assistência obstétrica (referência modelo Boas Práticas) segundo as variáveis socioeconômicas, demográficas, obstétricas, da assistência pré-natal, das instituições e da atuação dos profissionais de saúde em gestantes de risco habitual. Região Sul do Brasil, 2011 ( $n=2.668)$.

\begin{tabular}{|c|c|c|c|c|}
\hline & \multicolumn{4}{|c|}{ Modelo de assistência obstétrica } \\
\hline & \multicolumn{2}{|c|}{ Intervencionista I } & \multicolumn{2}{|c|}{ Intervencionista II } \\
\hline & $\begin{array}{l}\text { OR ajustada * } \\
\text { (IC95\%) }\end{array}$ & Valor de $p * *$ & $\begin{array}{l}\text { OR ajustada * } \\
\quad \text { (IC95\%) }\end{array}$ & Valor de $p * *$ \\
\hline \multicolumn{5}{|l|}{ Idade (anos) } \\
\hline $12-19$ & $0,94(0,65-1,35)$ & 0,723 & $0,71(0,46-1,10)$ & 0,123 \\
\hline \multicolumn{5}{|l|}{$20-34$} \\
\hline 35 ou mais & $1,11(0,79-1,55)$ & 0,556 & $1,25(0,80-1,95)$ & 0,314 \\
\hline \multicolumn{5}{|l|}{ Escolaridade } \\
\hline \multicolumn{5}{|l|}{ Ensino Fundamental incompleto } \\
\hline Ensino Fundamental completo & $0,95(0,74-1,22)$ & 0,666 & $2,60(1,55-4,37)$ & 0,001 \\
\hline Ensino Médio completo & $1,00(0,72-1,38)$ & 0,986 & $8,78(4,89-15,75)$ & $<0,001$ \\
\hline Ensino Superior completo & $0,66(0,34-1,27)$ & 0,206 & $18,72(8,43-41,59)$ & $<0,001$ \\
\hline \multicolumn{5}{|l|}{ Classe ABIPEME } \\
\hline \multicolumn{5}{|l|}{$\mathrm{D} / \mathrm{E}$} \\
\hline C & $1,27(0,85-1,91)$ & 0,237 & $1,88(0,64-5,50)$ & 0,245 \\
\hline$A / B$ & $1,32(0,77-2,26)$ & 0,313 & $3,17(0,70-14,26)$ & 0,129 \\
\hline \multicolumn{5}{|l|}{ Paridade } \\
\hline \multicolumn{5}{|l|}{$\geq 1$} \\
\hline 0 & $0,97(0,71-1,31)$ & 0,835 & $1,11(0,84-1,47)$ & 0,451 \\
\hline \multicolumn{5}{|l|}{ Idade gestacional (semanas) } \\
\hline $37-38$ & $1,12(0,84-1,49)$ & 0,438 & $1,63(1,16-2,29)$ & 0,006 \\
\hline \multicolumn{5}{|l|}{$39-40$} \\
\hline 41 & $0,82(0,59-1,13)$ & 0,223 & $0,24(0,16-0,36)$ & $<0,001$ \\
\hline \multicolumn{5}{|l|}{ Local de realização do pré-natal } \\
\hline \multicolumn{5}{|l|}{ Serviço público } \\
\hline Serviço particular ou plano de saúde & $0,84(0,49-1,44)$ & 0,513 & $13,88(2,60-73,97)$ & 0,003 \\
\hline Serviço público e particular & $0,60(0,33-1,10)$ & 0,098 & $2,74(0,52-14,31)$ & 0,226 \\
\hline \multicolumn{5}{|l|}{$\begin{array}{l}\text { Profissional que atendeu a maior parte das } \\
\text { consultas de pré-natal }\end{array}$} \\
\hline \multicolumn{5}{|l|}{ Enfermeiro } \\
\hline Médico & $2,29(1,05-4,97)$ & 0,037 & $17,81(5,06-62,70)$ & $<0,001$ \\
\hline \multicolumn{5}{|l|}{ Preferência inicial pela via de parto } \\
\hline \multicolumn{5}{|l|}{ Parto vaginal } \\
\hline Cesárea & $1,20(0,89-1,63)$ & 0,230 & $2,55(1,72-3,77)$ & $<0,001$ \\
\hline Não tinha preferência & $1,00(0,49-2,07)$ & 0,993 & $0,96(0,42-2,21)$ & 0,928 \\
\hline \multicolumn{5}{|l|}{$\begin{array}{l}\text { Aconselhamento sobre o tipo de parto no } \\
\text { pré-natal }\end{array}$} \\
\hline \multicolumn{5}{|l|}{ Parto vaginal é mais seguro } \\
\hline Cesárea é mais segura & $1,17(0,74-1,84)$ & 0,494 & $2,61(1,49-4,55)$ & 0,001 \\
\hline Ambos são seguros & $0,85(0,62-1,17)$ & 0,307 & $1,75(1,11-2,78)$ & 0,018 \\
\hline Não ficou esclarecida & $1,09(0,59-2,02)$ & 0,774 & $1,87(1,02-3,42)$ & 0,042 \\
\hline \multicolumn{5}{|c|}{ Decisão pela via de parto no final da gestação } \\
\hline \multicolumn{5}{|l|}{ Parto vaginal } \\
\hline Cesárea & $1,77(1,14-2,75)$ & 0,012 & $7,23(4,08-12,81)$ & $<0,001$ \\
\hline Não tinha preferência & $1,18(0,70-2,02)$ & 0,526 & $0,94(0,56-1,59)$ & 0,813 \\
\hline
\end{tabular}

(continua) 


\begin{tabular}{|c|c|c|c|c|}
\hline & \multicolumn{4}{|c|}{ Modelo de assistência obstétrica } \\
\hline & \multicolumn{2}{|c|}{ Intervencionista I } & \multicolumn{2}{|c|}{ Intervencionista II } \\
\hline & $\begin{array}{l}\text { OR ajustada * } \\
\text { (IC95\%) }\end{array}$ & Valor de $p * *$ & $\begin{array}{l}\text { OR ajustada * } \\
\text { (IC95\%) }\end{array}$ & Valor de $p$ ** \\
\hline \multicolumn{5}{|c|}{ Fonte de pagamento do nascimento } \\
\hline \multicolumn{5}{|l|}{ Pública } \\
\hline Privada & $1,18(0,48-2,89)$ & 0,718 & $66,99(7,61-589,73)$ & $<0,001$ \\
\hline \multicolumn{5}{|c|}{ Mesmo profissional no pré-natal e nascimento } \\
\hline Sim & $1,14(0,54-2,40)$ & 0,732 & $9,63(3,61-25,70)$ & $<0,001$ \\
\hline
\end{tabular}

ABIPEME: Associação Brasileira de Institutos de Pesquisa de Mercado; IC95\%: intervalo de 95\% de confiança.

Nota: destacados em negrito valores de $p<0,05$.

* OR ajustada para idade, escolaridade, classe econômica ABIPEME e primiparidade;

** Nível de significância pelo Teste de Wald.

trabalho de parto, ou parto. Ademais, a decisão por estudar mulheres classificadas como gestantes de risco habitual justifica-se pela inexistência de fatores de risco obstétricos ou prévios que pudessem indicar a realização ou não das práticas obstétricas estudadas.

Como resultado do agrupamento das práticas obstétricas, o modelo denominado Boas Práticas apresentou as maiores proporções de práticas baseadas em evidência e a maior aproximação do conceito de humanização da assistência ao nascimento. Já o modelo Intervencionista I sugere uma intencionalidade pelo parto vaginal como resultado final, mas que não valoriza a realização de práticas que promovam apoio e suporte à mulher ou bem-estar ao recém-nascido. Por sua vez, o modelo Intervencionista II, com a maior proporção de práticas obstétricas intervencionistas, utiliza abusivamente o uso da tecnologia, mas garante a presença do acompanhante no nascimento.

Já na análise múltipla, foi observado que as desigualdades sociais no acesso ao financiamento público ou privado da assistência à saúde permearam a formação dos modelos de assistência obstétrica. Também foi atestado que a assistência pública à saúde apresenta diferentes contextos. Um deles é alicerçado nos programas públicos e oferece melhor qualidade. Um segundo contexto demonstra preocupações com os elevados percentuais de cesárea, mas não considera os princípios de humanização da assistência. Com relação à assistência privada, atestou-se que é padronizada e centrada no profissional médico, com maiores níveis de intervenção. Por último, comprovou-se que fatores culturais e a atuação dos profissionais de saúde estiveram intimamente relacionados aos modelos de assistência obstétrica identificados.

No modelo de assistência obstétrica Boas Práticas, foi possível constatar o atendimento pelo sistema público de saúde e demais características, que se assemelharam ao que preconizam as políticas e os programas do Ministério da Saúde brasileiro. No serviço público de saúde, o acompanhamento pré-natal incorpora a participação de profissionais médicos e enfermeiros em consultas subsequentes, com avaliação periódica da classificação de risco gestacional e, quando indicado, o encaminhamento para o serviço de referência. Dentre as atividades para uma assistência pré-natal efetiva, são descritas a preparação e o incentivo ao parto normal. Quanto a esse ponto, observam-se campanhas publicitárias para a conscientização sobre seus benefícios e, mais atualmente, campanhas para o esclarecimento das condutas e procedimentos benéficos ou procedimentos que não devem ser realizados de forma rotineira na assistência obstétrica 12,13 .

São ainda características compatíveis com o modelo com Boas Práticas as orientações provenientes de diretrizes internacionais para a assistência obstétrica, o acesso à informação de qualidade durante o período gestacional que busca o empoderamento das mulheres para uma vivência plena desse processo e uma conscientização para a escolha do melhor tratamento 2. Assim, há uma maior compreensão das mulheres, a partir de informações transmitidas durante o pré-natal, de que o parto vaginal é mais seguro para a mãe. Além disso, é possível tomar uma decisão final pelo parto vaginal, na ausência de indicações clínicas para uma cesárea, a julgar os riscos a que estão expostas as mulheres submetidas a esse tipo de cirurgia 10 . 
Tabela 4

Desfechos maternos (percepção da mulher sobre respeito, privacidade, clareza nas explicações, tempo disponível para fazer perguntas, participação nas decisões e satisfação geral com o atendimento) e neonatais (manobras de reanimação, internação hospitalar e alta em aleitamento materno exclusivo) segundo modelo de assistência obstétrica em gestantes de risco habitual. Região Sul do Brasil, 2011.

\begin{tabular}{|c|c|c|c|}
\hline & $\%(I C 95 \%)$ & OR ajustada * (IC95\%) & Valor de $p$ ** \\
\hline Respeito dos profissionais $(n=2.235$ ) & $88,7(85,7-91,1)$ & & \\
\hline Boas Práticas & $90,4(86,9-93,1)$ & & \\
\hline Intervencionista I & $83,9(79,1-87,7)$ & $0,55(0,34-0,88)$ & 0,015 \\
\hline Intervencionista II & $95,8(93,5-97,2)$ & $1,84(1,03-3,30)$ & 0,040 \\
\hline Privacidade no trabalho de parto e nascimento $(n=2.235)$ & $92,7(90,7-94,3)$ & & \\
\hline Boas Práticas & $93,0(90,4-94,9)$ & & \\
\hline Intervencionista I & $90,4(86,5-93,2)$ & $0,71(0,42-1,18)$ & 0,181 \\
\hline Intervencionista II & $97,3(95,8-98,3)$ & $1,94(1,10-3,41)$ & 0,022 \\
\hline Clareza nas explicações $(n=2.235)$ & $86,3(83,8-88,5)$ & & \\
\hline Boas Práticas & $85,5(81,5-88,8)$ & & \\
\hline Intervencionista I & $83,6(79,7-86,9)$ & $0,88(0,59-1,31)$ & 0,518 \\
\hline Intervencionista II & $93,5(90,8-95,4)$ & $2,00(1,27-3,16)$ & 0,004 \\
\hline Tempo disponível para fazer perguntas $(n=2.235)$ & $85,8(83,1-88,1)$ & & \\
\hline Boas Práticas & $85,5(82,4-88,1)$ & & \\
\hline Intervencionista | & $82,3(77,7-86,2)$ & $0,80(0,54-1,16)$ & 0,234 \\
\hline Intervencionista II & $93,8(91,0-95,7)$ & $2,16(1,42-3,28)$ & 0,001 \\
\hline Participação nas decisões $(n=2.235$ ) & $87,5(85,1-89,6)$ & & \\
\hline Boas Práticas & $87,7(83,6-90,9)$ & & \\
\hline Intervencionista I & $84,0(80,5-86,9)$ & $0,75(0,49-1,14)$ & 0,173 \\
\hline Intervencionista |I & $94,9(91,8-96,9)$ & $2,78(1,53-5,07)$ & 0,001 \\
\hline Satisfação geral $(n=2.235)$ & $92,3(90,5-93,8)$ & & \\
\hline Boas Práticas & $91,4(88,8-93,4)$ & & \\
\hline Intervencionista I & $90,6(87,6-92,9)$ & $0,88(0,58-1,34)$ & 0,548 \\
\hline Intervencionista II & $97,9(96,3-98,8)$ & $2,96(1,56-5,65)$ & 0,001 \\
\hline Manobras de reanimação $(n=2.648)$ & $10,8(8,1-4,3)$ & & \\
\hline Boas Práticas & $6,4(4,5-9,0)$ & & \\
\hline Intervencionista I & $12,1(7,7-18,5)$ & $2,02(1,08-3,78)$ & 0,029 \\
\hline Intervencionista || & $16,4(9,4-26,8)$ & $2,83(1,34-5,97)$ & 0,007 \\
\hline Internação hospitalar ( $\mathrm{n}=2.648$ ) & $4,7(3,6-6,1)$ & & \\
\hline Boas Práticas & $6,3(4,2-5,8)$ & & \\
\hline Intervencionista I & $4,2(3,1-5,8)$ & $0,66(0,38-1,12)$ & 0,119 \\
\hline Intervencionista II & $2,8(1,6-4,7)$ & $0,47(0,22-1,02)$ & 0,057 \\
\hline Alta em aleitamento materno exclusivo $(n=2.228)$ & $94,0(91,7-95,6)$ & & \\
\hline Boas Práticas & $96,2(93,4-96,1)$ & & \\
\hline Intervencionista I & $93,3(88,6-96,1)$ & $0,53(0,24-1,17)$ & 0,112 \\
\hline Intervencionista II & $91,2(88,3-93,4)$ & $0,51(0,26-0,99)$ & 0,049 \\
\hline
\end{tabular}

ABIPEME: Associação Brasileira de Institutos de Pesquisa de Mercado; IC95\%: intervalo de 95\% de confiança; OR: odds ratio.

Nota: destacados em negrito valores de $p<0,05$.

* OR ajustada para idade, escolaridade, classe econômica ABIPEME e primiparidade;

** Nível de significância pelo Teste de Wald.

No modelo de assistência obstétrica Intervencionista I, observa-se a outra face na dualidade da assistência prestada pelo sistema público de saúde. Diferenças encontradas na formação dos modelos (diferença na proporção de práticas por hospital) ou na ausência de associações, tanto na comparação do financiamento da assistência quanto nas características socioeconômicas e demográficas da clientela entre os modelos com Boas Práticas e Intervencionista I, confirmam essa afirmação. Esses resultados ratificam a dificuldade na implementação dos programas públicos e a adequação dos serviços de saúde, quer com práticas institucionais enraizadas, quer com profissionais tecnocráticos inseridos 
nas instituições que desconhecem ou ignoram os benefícios de uma prática baseada em evidência ou fundamentada nos princípios de humanização.

À vista dos resultados desses dois modelos, compreende-se que as mulheres com menor nível de escolaridade ora possuem acesso a mais alta qualidade da assistência (modelo com Boas Práticas), ora são submetidas a assistência intervencionista e desumanizada (modelo Intervencionista II).

No modelo Intervencionista II, observa-se o predomínio da assistência privada à saúde, com características distintas dos dois outros modelos. Surgem fatores culturais, como a maior chance da preferência inicial pela cesárea, e essa preferência não é desconstruída durante o pré-natal. Ao contrário, torna-se consolidada na percepção das mulheres como uma via de parto segura. Esse aspecto levanta questionamentos sobre a qualidade das informações transmitidas e a influência dos profissionais, pois não foram encontradas diferenças na preferência inicial pela via de parto, na análise descritiva das mulheres incluídas nesse modelo. A interpretação de estudos anteriores apoia esse questionamento de que as mulheres confiam nas informações fornecidas durante o pré-natal e acreditam que as indicações para a realização de uma cesárea resultaram em maior segurança, mesmo ultrapassando a frequência de valores aceitáveis 14 .

Soma-se a isso a constituição de um modelo com mulheres de maior escolaridade e que, por esse motivo, deveriam ter maior acesso à informação de qualidade e à contestação baseada em cientificidade por parte dos profissionais, para a não adoção das melhores práticas, ou seja, a decisão por uma cesárea 15. Ademais, deve-se destacar o caso das mulheres que foram acompanhadas no pré-natal por profissional médico e que tiveram a continuidade do atendimento no momento do nascimento, característica que promove uma relação de confiança. Contudo, a continuidade no atendimento pelo mesmo profissional neste estudo, implicou na realização de um procedimento cirúrgico com data e hora marcada, evidenciado pela baixa prevalência de mulheres que entraram em trabalho de parto e pela realização da cesárea antes da 39ª semana de gestação, aspectos associados com maior chance de resultados adversos neonatais 16 .

Por conseguinte, uma assistência com a falsa pretensão de humanização e segurança, que respalda e fortalece a decisão final pela cesárea, possibilita a presença do acompanhante, mas não considera os riscos a que estão sendo expostos mulheres e recém-nascidos. Além disso, são práticas que comprometem a vida reprodutiva futura dessas mulheres, pelo maior risco de complicações placentárias, em gestações subsequentes 17 .

Os resultados encontrados no modelo de assistência obstétrica Intervencionista II endossam uma assistência padronizada e centrada no profissional médico. Trata-se de profissionais que possuem formação desenvolvida em hospitais de referência, com maior nível de complexidade para o atendimento de situações que realmente necessitam de intervenções. Essas características podem limitar a formação e a capacitação para o acompanhamento do trabalho de parto e parto, na perspectiva de uma assistência obstétrica fisiológica 18 . Nesse contexto, tem-se a atuação de enfermeiras obstetras, obstetrizes e midwives na atenção ao parto, profissionais com formação específica para o atendimento de mulheres sem complicações ${ }^{19}$, mas que encontram pouco espaço ou autonomia, em uma assistência excessivamente medicalizada 20. Tais profissionais realizam comprovadamente um menor número de intervenções na assistência ao trabalho de parto e parto 19,21,22, com maiores níveis de satisfação dos sujeitos envolvidos 22 .

A Agência Nacional de Saúde Suplementar (ANS) envida esforços para a reversão das elevadas taxas de cesárea nesse setor, com a implementação do que chama modelos de atenção ao parto alternativos (hospital atípico) 23. As características do hospital típico do setor de saúde suplementar são semelhantes ao modelo Intervencionista II identificado. No hospital atípico, com diferentes equipes no pré-natal e na atenção ao parto, o trabalho colaborativo é realizado entre enfermeiras obstetras e médicos, e o pagamento dos profissionais é feito por tempo de serviço, independente do número de procedimentos realizados. A pesquisa mostrou menor prevalência de cesáreas nos hospitais atípicos $(47,8 \%$ versus $90,8 \%) 24$.

No modelo Intervencionista II, as mulheres apresentaram maior satisfação com o atendimento recebido, apesar dos maiores índices de intervenções obstétricas, na comparação com o modelo com Boas Práticas. São associações condizentes com estudos sobre satisfação, nos quais seus resultados são produtos da expectativa em relação ao atendimento, independente da qualidade dos serviços prestados 25 . Ademais, a interrupção da gestação em mulheres que não entraram em trabalho de parto 
pode ter contribuído para esse resultado 11 , pelo menor tempo de internação e menor contato com a equipe de saúde.

A percepção de violência verbal, psicológica e física esteve presente, independente do modelo de assistência obstétrica identificado, e representa a desumanização da assistência e a perpetuação da opressão feminina na assistência à saúde 26 . É uma violência que vai além das contextualizações supracitadas, pois engloba a realização de práticas obstétricas desnecessárias e por vezes danosas 27,28.

$\mathrm{O}$ resultado desse estudo acerca da satisfação das mulheres esteve associado à continuidade na assistência, corroborando outras investigações 22,23,24,25, porém não garantiu os melhores resultados em saúde. No modelo Intervencionista II, a chance de reanimação do recém-nascido em sala de parto mais que dobrou em relação ao modelo com Boas Práticas. Os benefícios do aleitamento materno exclusivo, prática que demanda conhecimento, habilidade e apoio dos profissionais envolvidos 29 , tiveram menos chance no modelo Intervencionista II, na comparação com o modelo com Boas Práticas.

As associações encontradas nos modelos de assistência obstétrica, de acordo com a fonte de pagamento pública ou privada, mostraram desigualdades sociais de acesso que não se mantiveram na análise da classe econômica. Esse dado leva à compreensão de que as mulheres, independente da classe econômica, buscam serviços de qualidade, reconhecidos nos preceitos de humanização da assistência obstétrica, como é o modelo com Boas Práticas. E o que leva as mulheres para o atendimento no modelo Intervencionista II? Na fuga do parto vaginal traumático ofertado no modelo Intervencionista I, a solução pode estar no agendamento de uma cesárea. Esse fato foi identificado em estudo anterior, em que mulheres de classe social menos favorecida direcionaram a renda familiar para os serviços medicalizados, na busca por cesárea 30 .

O desenvolvimento deste estudo não elucida todos os motivos que justificam o atendimento em determinado modelo de assistência. Há fatores que estão além das características identificadas e que contemplam questões de ordem política, cultural, institucional e organizacional, com destaque nesta última, para a remuneração dos profissionais de saúde e os incentivos de produtividade 31.

A baixa prevalência do trabalho de parto foi uma das limitações do estudo, o que impossibilitou a verificação de associações entre as práticas durante o trabalho de parto e os modelos de assistência obstétrica a serem identificados.

Observa-se, na Região Sul do Brasil, o predomínio de uma assistência obstétrica na contramão das melhores evidências, com praticamente sete em cada dez mulheres atendidas nos modelos de assistência obstétrica Intervencionista I e II. Mesmo no modelo com Boas Práticas, há persistência de elevadas taxas de cesárea. E o que se pode fazer para mudar essa realidade? $\mathrm{O}$ fato é que as mulheres precisam de uma escolha informada, e não de um consentimento informado, pois as evidências científicas acumuladas sobre a prática obstétrica humanizada não foram suficientes para modificar a atitude dos profissionais ou a reestruturação dos serviços de saúde 31 . Além disso, as mulheres não devem se sentir coagidas para a realização de um procedimento cirúrgico, pelo simples fato de não se sentirem seguras ou pela falta de atendimento profissional adequado para a vivência de um trabalho de parto e nascimento humanizados 32 .

Conclui-se, a partir deste estudo, que a mulheres assistidas em hospitais públicos possuem mais chance de serem beneficiadas com as melhores práticas. Outra conclusão é que o acompanhamento das mulheres durante o pré-natal tem impacto sobre a decisão pela via de parto e o atendimento prestado no momento do nascimento. Acrescenta-se que nem o vínculo construído durante o pré-natal nem a assistência privada à saúde asseguram um melhor atendimento obstétrico. Isso significa que o modelo de assistência à saúde que prevalece na iniciativa privada encontra-se distorcido na visão de segurança das mulheres e não possibilita nem ao menos a experiência do desencadeamento do trabalho de parto.

É urgente a necessidade de mudança na estrutura dos serviços de saúde, principalmente no atendimento às gestantes de risco habitual, mulheres que necessitam de acompanhamento, cuidado, apoio, e não intervenção. Há necessidade de práticas educativas direcionadas aos profissionais que prestam assistência, para redução das intervenções e consequente melhora dos desfechos maternos e neonatais 33. A exemplo de países desenvolvidos 22 e de pesquisas nacionais 19,21,24, o Brasil necessita de maior inserção e atuação de enfermeiras obstetras e obstetrizes, na assistência prestada durante a gestação e o parto, dentro ou fora de instituições hospitalares. 


\section{Colaboradores}

M. B. Velho, O. M. Brüggemann, C. McCourt, S. G. N. Gama, R. Knobel, A. C. Gonçalves e E. d'Orsi contribuíram na concepção e projeto ou análise e interpretação dos dados; redação do artigo ou revisão crítica relevante do conteúdo intelectual; e aprovação final da versão a ser publicada, com responsabilidade sobre todos os aspectos do trabalho, na garantia da exatidão e integridade de qualquer parte da obra.

\section{Informações adicionais}

ORCID: Manuela Beatriz Velho (0000-0001-66606978); Odaléa Maria Brüggemann (0000-00031210-6040); Christine McCourt (0000-00034765-5795); Silvana Granado Nogueira da Gama (0000-0002-9200-0387); Roxana Knobel (00000001-9180-4685); Annelise de Carvalho Gonçalves (0000-0002-8770-0653); Eleonora d'Orsi (00000003-2027-1089).

\section{Agradecimentos}

Este artigo foi realizado com dados da pesquisa Nascer no Brasil e obteve financiamento dos seguintes órgãos: Conselho Nacional de Desenvolvimento Científico e Tecnológico (CNPq); Departamento de Ciência e Tecnologia, da Secretaria de Ciência, Tecnologia e Insumos Estratégicos do Ministério da Saúde; Escola Nacional de Saúde Pública Sérgio Arouca, Fundação Oswaldo Cruz (Projeto INOVA); Fundação de Amparo à Pesquisa do Estado do Rio de Janeiro (Faperj). Também contou com apoio financeiro do CNPq na forma de bolsa de doutorado sanduíche no exterior para M. B. Velho e bolsa de produtividade em pesquisa para O. M. Brüggemann e E. d'Orsi.

\section{Referências}

1. Melnyk BM, Fineout-Overholt E. Evidencebased practice in nursing \& healthcare: a guide to best practice. 2nd Ed. Philadelphia: Lippincott Williams \& Wilkins; 2010.

2. National Institute for Health and Care Excellence. Antenatal care for uncomplicated pregnancies. Clinical guideline. London: National Institute for Health and Care Excellence; 2016.

3. World Health Organization. Care in normal birth: a practical guide. Geneva: World Health Organization; 1996.

4. World Health Organization. World Health Organization recommendations: intrapartum care for a positive childbirth experience. Geneva: World Health Organization; 2018.

5. Patah LEM, Malik AM. Models of childbirth care and cesarean rates in different countries. Rev Saúde Pública 2011; 45:185-94.

6. Vasconcellos MTLD, Silva PLN, Pereira APE, Schilithz AOC, Souza Junior PRBS, Szwarcwald CL. Desenho da amostra Nascer no Brasil: Pesquisa Nacional sobre Parto e Nascimento. Cad Saúde Pública 2014; 30 Suppl:S49-58.

7. Leal MDC, Silva AAM, Dias MAB, Gama SGN, Rattner D, Moreira ME, et al. Birth in Brazil: national survey into labour and birth. Reprod Health 2012; 9:15.

8. Dahlen HG, Tracy S, Tracy M, Bisits A, Brown C, Thornton C. Rates of obstetric intervention among low-risk women giving birth in private and public hospitals in NSW: a population-based descriptive study. BMJ Open 2012; 2:e001723.

9. Leal MC, Pereira APE, Domingues RMSM, Theme Filha MM, Dias MAB, Nakamura-Pereira $\mathrm{M}$, et al. Intervenções obstétricas durante o trabalho de parto e parto em mulheres brasileiras de risco habitual. Cad Saúde Pública 2014; 30 Suppl:S17-47.

10. Villar J, Carroli G, Zavaleta N, Donner A, Wojdyla D, Faundes A, et al. Maternal and neonatal individual risks and benefits associated with caesarean delivery: multicentre prospective study. BMJ 2007; 335:1025.

11. d'Orsi E, Brüggemann OM, Diniz CSM, Aguiar JM, Gusman CR, Torres JA, et al. Desigualdades sociais e satisfação das mulheres com o atendimento ao parto no Brasil: estudo nacional de base hospitalar. Cad Saúde Pública 2014; 30 Suppl:S154-68.

12. Ministério da Saúde; Instituto Sírio-Libanês de Ensino e Pesquisa. Protocolos da atenção básica: saúde das mulheres. Brasília: Ministério da Saúde; 2016.

13. Ministério da Saúde. Promoção da Saúde SUS. Incentivo ao parto normal. Brasília: Ministério da Saúde; 2016.

14. Potter JE, Berquó E, Perpétuo IHO, Leal OF, Hopkins K, Souza MR, et al. Unwanted caesarean sections among public and private patients in Brazil: prospective study. BMJ 2001; 323:1155-8. 
15. Alonso B, Silva FM, Latorre MR, Diniz C, Bick D. Caesarean birth rates in public and privately funded hospitals: a cross-sectional study. Rev Saúde Pública 2017; 51:101.

16. ACOG Committee Opinion No. 579: definition of term pregnancy. Obstet Gynecol 2013; 122:1139-40.

17. Hu HT, Xu JJ, Lin J, Li C, Wu IT, Sheng JZ, et al. Association between first caesarean delivery and adverse outcomes in subsequente pregnancy. BMC Pregnancy Childbirth 2018; 18:273.

18. Sass N, Whang SM. Dados epidemiológicos, evidências e reflexões sobre a indicação de cesárea no Brasil. Diagn Tratamento 2009; 14:133-7.

19. Gama SGN, Viellas EF, Torres JA, Bastos MH, Brüggemann OM, Theme Filha MM, et al. Labor and birth care by nurse with midwifery skills in Brazil. Reprod Health 2016; 13 Suppl 3:123.

20. Sanches NC, Mamede FV, Vivancos RBZ. The profile of women who have experienced cesarean section and obstetric care at a public maternity hospital in Ribeirao Preto. Texto \& Contexto Enferm 2012; 21:418-26.

21. Vogt SE, Diniz SG, Tavares CM, Santos NCP, Schneck CA, Zorzam B, et al. Características da assistência ao trabalho de parto e parto em três modelos de atenção no SUS, no Município de Belo Horizonte, Minas Gerais, Brasil. Cad Saúde Pública 2011; 27:1789-800.

22. Sandall J, Soltani H, Gates S, Devane D. Midwife-led continuity models versus other models of care for childbearing women. Cochrane Database Syst Rev 2016; (4):CD004667.

23. Agência Nacional de Saúde Suplementar. O modelo de atenção obstétrica no setor de Saúde Suplementar no Brasil: cenários e perspectivas. Rio de Janeiro: Agência Nacional de Saúde Suplementar; 2008.

24. Torres JA, Domingues RMSM, Sandall J, Hartz Z, Gama SGN, Theme Filha MM, et al. Cesariana e resultados neonatais em hospitais privados no Brasil: estudo comparativo de dois diferentes modelos de atenção perinatal. Cad Saúde Pública 2014; 30 Suppl:S220-31.
25. Espiridião MA, Trad LAB. Avaliação de satisfação de usuários: considerações teórico-conceituais. Cad Saúde Pública 2006; 22:1267-76.

26. Souza JP, Pileggi-Castro C. Sobre o parto e o nascer: a importância da prevenção quaternária. Cad Saúde Pública 2014; 30 Suppl:S11-3.

27. Tesser CD, Knobel R, Andrezzo HFA, Diniz SG. Violência obstétrica e prevenção quaternária: o que é e o que fazer. Rev Bras Med Fam Comunidade 2015; 10:1-12.

28. Marrero L, Brüggemann OM. Institutional violence during the parturition process in $\mathrm{Bra}-$ zil: integrative review. Rev Bras Enferm 2018; 71:1152-61.

29. Departamento de Ações Programáticas e Estratégicas, Secretaria de Atenção à Saúde, Ministério da Saúde. Atenção à saúde do recémnascido: guia para os profissionais de saúde. Brasília: Ministério da Saúde; 2011.

30. Béhague DP, Victora CG, Barros FC. Consumer demand for caesarean sections in Brazil: informed decision making, patient choice, or social inequality? A population based birth cohort study linking ethnographic and epidemiological methods. BMJ 2002; 324:942-5.

31. Maia MB. Humanização do parto: política pública, comportamento organizacional e ethos profissional na rede hospitalar pública e privada de Belo Horizonte [Dissertação de Mestrado]. Belo Horizonte: Pontifícia Universidade Católica; 2008.

32. Leone P, Padmadas SS, Matthews S. Community factors affecting rising caesarean section rates in developing countries: an analysis of six countries. Soc Sci Med 2008; 67:1236-46.

33. Côrtes CT, Oliveira SMJV, Santos RCS, Francisco AA, Riesco MLG, Shimoda GT. Implementation of evidence-based practices in normal delivery care. Rev Latinoam Enferm 2018; 26:e2988. 


\section{Abstract}

The study sought to identify obstetric care models for low-risk pregnancies in the Southern Region of Brazil and to estimate factors associated with these models and maternal and neonatal outcomes. This is a cross-sectional, hospital-based study using data from the Birth in Brazil survey regarding puerperae and newborns. We identified 2,668 lowrisk pregnant women. We carried out an exploratory analysis using the proportion of practices per hospital, among them inducing labor, presence of a companion, cesarean section and skin-to-skin contact, in order to obtain the care models we called Best Practice, Interventionist I and Interventionist II. We then carried out an inferential analysis of the associated characteristics. Results show that access to public or private funding, cultural factors and actions taken by health professional are associated with the care models. Public care had different contexts, one based on public policies and evidence-based practices; and another, that suggests the intentionality of vaginal delivery without considering humanization principles. Private care, on the other hand, is standardized and centered on the medical professional, with higher intervention levels. We conclude there is a predominance of interventionist obstetric care models in the Southern Region of Brazil, a type of care that goes against the best evidence, and that women who receive care in public hospitals have greater chances of benefiting from good practices.

Obstetric Labor; Cesarean Section; Perinatal Care; Professional Practice; Evidence-Based Practice

\section{Resumen}

Los objetivos del estudio fueron identificar modelos de asistencia obstétrica en gestantes de riesgo habitual en la región sur de Brasil, estimar los factores asociados a estos modelos y los desenlaces maternos y neonatales. Es un estudio transversal, a partir de la pesquisa Nascer no Brasil, de base hospitalaria, compuesta por puérperas y recién nacidos. Se identificaron a 2.668 gestantes de riesgo habitual. Se procedió a un análisis exploratorio utilizando la proporción de prácticas por hospital, entre ellas el desencadenamiento de la labor de parto, presencia de acompañante, cesárea y contacto piel a piel, para la obtención de modelos de asistencia obstétrica, denominados Buenas Prácticas, Intervencionista I, e Intervencionista II; seguido de un análisis inferencial de las características asociadas. Los resultados mostraron que el acceso a la financiación pública o privada, factores culturales y la actuación de los profesionales de salud presentaron asociaciones con los modelos de asistencia. La asistencia pública presentó diferentes contextos, el primero basado en políticas públicas y en la práctica fundamentada en la evidencia; y un segundo, que sugiere la intencionalidad del parto vaginal sin considerar los principios de humanización; mientras que la asistencia privada está estandarizada y centrada en el profesional médico, con mayores niveles de intervención. Se concluye que existe un predominio de los modelos de asistencia obstétrica intervencionistas en la región sur de Brasil, una asistencia a contracorriente de las mejores evidencias, así como que las mujeres asistidas en hospitales públicos tienen una mayor oportunidad de beneficiarse de las buenas prácticas.

Trabajo de Parto; Cesárea; Atención Perinatal; Práctica Profesional; Práctica Clínica Basada en la Evidencia
Recebido em 10/Mai/2018

Versão final reapresentada em 09/Out/2018

Aprovado em 29/Nov/2018 